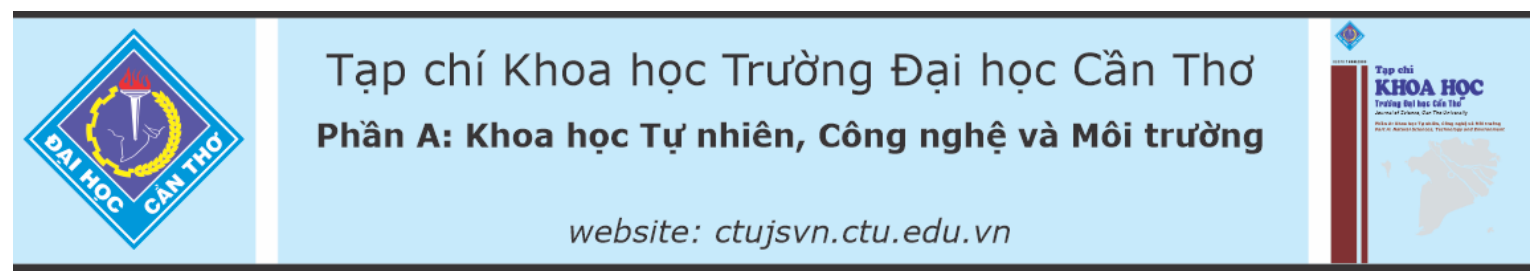

DOI:10.22144/ctu.jvn.2021.167

\title{
KIỂM NGHIỆM KHẢ NĂNG KẾT HỢP GIŨ̉A ĐIỀU KHIỂN PI VÀ TRƯợT THÍCH NGHI TRÊN THIẾT BỊ GUNT-RT020
}

\author{
Lê Tấn Mỹ, Trần Xa Lil, Trần Thanh Hùng và Nguyễn Chí Ngôn* \\ Khoa Công nghệ, Truờng Đại hoc Cần Tho \\ "Nguò̀i chịu trách nhiệm về bài viết: Nguyễn ChíNgôn (email: ncngon@ctu.edu.vn)
}

\section{Thông tin chung:}

Ngày nhận bài: 13/07/2021

Ngày nhận bài sủa: 13/08/2021

Ngày duyệt đăng: 25/12/2021

\section{Title:}

Testing the combination of the $P I$ and adaptive sliding mode control on the GUNT-RTO2O Device

\section{Tù khóa:}

Điều khiển truợt thich nghi, điều khiển lưu luợng chất lỏng, điều khiển PI, mạng neuron $R B F$

\section{Keywords:}

Adaptive Sliding Mode Control, Liquid flow control, PI controller, RBF neural network

\begin{abstract}
Although widely used in industry, with fixed parameters, the proportional integral (PI) controller is difficult to adapt to real conditions. Meanwhile, the sliding mode control (SMC) gives stable responses on nonlinear systems, but it has some limitations. This paper aims to propose a solution to combine the $P I$ control and the radial basis function $(R B F)$ neural network-based adaptive $S M C$, called PI-SMC control. The principle of this combination is to take SMC advantages to overcome the limitations of the PI controller and use the PI controller to push the sliding surface rapidly converge. The PI-SMC controller is tested on the liquid flow control device RTO2O of the GuntHamburg. The results show that the initial values of the RBF neural network and the coefficient of sliding surface has a great influence on the control quality. The experiment also shows that the adaptive sliding mechanism can overcome the limitation of fixed PI controller. With selected initial values, the PI-SMC controller has improved the flow response on the RTO20 with the overshoot is less than $5(\%)$; the settling time is less than $2(s)$; and the steadystate error is less than $0.3(\mathrm{l} / \mathrm{h})$.
\end{abstract}

\section{TÓM TẮT}

Mặc dù được sử dụng rộng rãi trong công nghiệp, nhung với tham số cố định, bộ điè̀u khiển tích phân tỷ lẹ PI (proportional integral controller) khó thich ưng với sự thay đổi của điều kiện thực tế. Trong khi đó, điều khiển truợt (sliding mode control - SMC) cho đáp ứng ổn định trên các đối tuợng phi tuyến, nhung lại tồn tại một số hạn chế. Bài báo này đề xuất giải pháp kể hợp giũa điều khiến PI và SMC thich nghi dụa trên mạng neuron hàm cơ sở xuyên tâm RBF (radial basis function neural network), goi tắt là điều khiển PI-SMC. Nguyên tắc kết hợp này là tận dụng uu điểm thích nghi, bền vũng của bộ SMC để khắc phục hạn chể của bộ điều khiển PI, đồng thời sủ dụng bộ PI mang năng luợng chủ đạo để đẩy bộ SMC nhanh chóng hội tu về mắt truợt. Bộ điều khiển PI-SMC được kiểm nghiệm trên thiết bị ổn định lư lượng RTO20 của hãng Gunt-Hamburg. Kết quả cũng cho giá trị khởi tạo của bộ RBF và hệ số mặt truợt ảnh hương lớn đến chất luợng điều khiển. Thự nghiệm cũng cho thấy cơ chế truợt thich nghi có thể khắc phục được hạn chế cố định tham số của bộ PI. Với giá trị khởi tạo của bộ tham số được chọn, bộ điều khiển PISMC đã cải thiện tốt đáp úng lưu lương trên hệ RT020 với độ vọt lố nhỏ hơn 5 (\%), thời gian xác lập nhỏ hơn 2 (giây) và sai số xác lập nhỏ hơn 0,3 (lit/giò). 


\section{GIỚI THIẸU}

Ra đời từ cuối thập niên 1960, kỹ thuật điều khiển trượt (Sliding Mode Control - SMC) được phát triển nhanh chóng và áp dụng nhiều trong các hệ thống tự động (Nguyễn Đức Minh, và ctv., 2009). Bộ điều khiển trượt sử dụng luật điều khiển chuyển đổi với tốc độ cao, để đưa hệ thống về mặt trượt và duy trì hệ thống trên mặt trượt đó. Uuu điểm của bộ điều khiển trượt là tính ổn định bền vững ngay cả khi hệ thống bị tác động của nhiễu hoặc tham số của mô hình thay đổi theo thời gian (Nguyễn Hoàng Dũng \& Dương Hoài Nghĩa, 2010; Nguyễn Hoàng Dũng, 2012). Điểm hạn chế cơ bản của SMC là cần phải biết chính xác mô hình của đối tượng khi thiết kế, nhưng điều này không phải lúc nào cũng đạt được. Bên cạnh đó, do luật trượt chuyển đổi với tốc độ cao, nên bộ SMC có thể gây ra hiện tượng dao động quanh mặt trượt (chattering).

Để giải quyết vấn đề khó xác định mô hình của đối tượng điều khiển trong thực tế, một số nghiên cứu đã sử dụng mạng neuron (Ye et al., 2020) để thay thế thành phần điều khiển tương đương trong luật trượt hoặc để xấp xỉ các thành phần bất định của hệ thống. Ngoài ra, để khắc phục hiện tượng dao động quanh mặt trượt, hàm dấu trong thành phần điều khiển bền vững cũng được nghiên cứu và thay thế bằng các hàm bão hòa hay các hàm dạng liên tục (Keshtkar et al., 2016). Thật vậy, Nguyễn Đức Minh và ctv. (2009) đã giới thiệu bộ SMC thích nghi dùng mạng neuron (ANSMC), áp dụng cho các đối tượng phi tuyến bất định hoặc không rõ mô hình. Kết quả mô phỏng trên hệ con lắc ngược cho thấy đã khắc phục được hiện tượng chattering. Nguyễn Hoàng Dũng \& Dương Hoài Nghĩa (2010) đã sử dụng mạng neuron hàm cơ sở xuyên tâm (RBFNN) để ước lượng trực tuyến các hàm phi tuyến trong luật điều khiển, đồng thời sử dụng logic mờ để ước lượng biên độ của luật điều khiển, dựa vào lý thuyết ổn định Lyapunov. Kết quả điều khiển được kiểm chứng bằng phần mềm MATLAB/Simulink cho thấy đáp ứng của bộ điều khiển có độ vọt lố thấp, thời gian xác lập được cải thiện. Bên cạnh đó, hàm trượt được thiết kế dựa trên bộ điều khiển Proportional Integral Derivative-PID cũng được áp dụng thành công trong việc kiểm soát các đối tượng phi tuyến (Nguyễn Hoàng Dũng, 2012). Giải pháp này được đề nghị nhằm giảm thiểu hiện tượng dao động quanh mặt trượt và giảm dao động trong tín hiệu điều khiển. Giải thuật được áp dụng cho hệ tay máy một bậc tự do. Kết quả mô phỏng trên MATLAB/Simulink cho thấy đáp ứng của hệ tay máy bám tốt tín hiệu tham khảo. Phương pháp SMC thích nghi dựa trên mạng neuron Radial Basic Function-RBF để kiểm soát hệ cầu cân bằng, cũng được xây dựng (Nguyễn Đình Tứ và ctv., 2017). Trong nghiên cứu này, tính bền vững của bộ điều khiển đã được đánh giá thông qua mô phỏng việc thay đổi tín hiệu tham khảo, khối lượng hòn bi và giả lập nhiễu cảm biến.

Điểm hạn chế của các nghiên cứu trên là chỉ dừng lại ở mức độ mô phỏng, mà chưa áp dụng trên mô hình thực tế. Bài báo này đề xuất kiểm nghiệm thực tế kỹ thuật SMC thích nghi (Miqoi et al., 2019) trên hệ ổn định lưu lượng chất lỏng - RT020 của hãng Gunt-Hamburg (Gunt Gerätebau $\mathrm{GmbH}$ [Gunt], 2004). Hệ RT020 được Gunt tích hợp sẵn bộ điều khiển tích phân tỷ lệ PI kinh điển. Bộ điều khiển PI với tham số cố định theo thời gian đã không còn đáp ứng tốt, do một số tham số của hệ RT020 đã thay đổi theo thời gian, đặc biệt là thời gian trễ của hệ thống. Việc kết hợp kỹ thuật điều khiển SMC thích nghi và bộ $\mathrm{PI}$ kinh điển trên hệ RT020 nhằm tận dụng khả năng thích nghi của bộ $\mathrm{SMC}$ để khắc phục hạn chế của bộ PI cố định. Mặc dù hệ RT020 khá tuyến, nhưng thời gian trễ là một yếu tố cần quan tâm và khó kiểm soát trong điều khiển (Gopika \& Latha, 2016). Đồng thời, công cụ giao tiếp giữa MATLAB và hệ RT020 thông qua card truyền thông Labjack (LabJack Corporation , 2003) đã được phát triển hoàn thiện bởi Nguyễn Chí Ngôn (2011), nên RT020 hoàn toàn thích hợp để kiểm chứng các giải thuật điều khiển.

Bộ điều khiển được xây dựng để kiểm nghiệm thực tế trong nghiên cứu này gồm 2 thành phần là Bộ điều khiển PI truyền thống và bộ SMC thích nghi dựa theo mạng neuron RBF. Hai bộ điều khiển này cùng tham gia quá trình kiểm soát hệ RT020. Khi có sự biến động của điều kiện thực tế, bộ điều khiển PI với tham số cố định có thể không đủ linh hoạt, thì bộ SMC thích nghi sẽ đảm nhận vai trò đó. Trong quá trình thiết kế bộ SMC thích nghi, một vài tham số của mặt trượt vẫn phải được xác định theo kinh nghiệm. Do đó, nghiên cứu này kỳ vọng thực nghiệm thực tế có thể cho phép khảo sát ảnh hưởng của việc lựa các tham số này, dựa theo các tiêu chuẩn chất lượng quen thuộc.

\section{PHƯƠNG PHÁP NGHIÊN CÚU}

\section{1. Đối tượng điều khiển}

Cấu trúc của hệ ổn định lưu lượng RT020 (Gunt, 2004) được trình bày trên Hình 1 . Chất lỏng trong bồn chứa (5) được bơm vào mạng điều tiết bằng máy bơm (4). Lưu lượng chất lỏng ở trong mạng có thể tăng hay giảm được bằng valve tiết lưu (2) hoặc thay 
đổi công suất máy bơm (4). Tình trạng hoạt động và các công tắc điều khiển được bố trí trên bảng (3). Lưu lượng chất lỏng trong mạng được đo bởi cảm biến (6), đồng thời biểu kiến bởi lưu lượng kế kiểu phao (1). Nhiệm vụ của bộ điều khiển là kiểm soát công suất của valve tiết lưu (2) để lưu lượng chất lỏng trong mạng được duy trì ở trạng thái ổn định mà người vận hành đặt trước, bù trừ được với biến đổi của tốc độ bơm. Thông số kỹ thuật của hệ RT020 được trình bày trong Bảng 1 .


Hình 1: Cấu trúc hệ ổn định lưu lượng RT020

1: luu lự̛ng kế kiểu phao, 2: valve tiết luu, 3: bảng hiển thị và điều khiển, 4: borm, 5: bồn chứa, 6: cảm biến lưu lương

Bảng 1: Một số thông số kỹ thuật của RT020

\begin{tabular}{lll}
\hline Đại lượng & Giá trị & Đơn vị \\
\hline Dung tích bồn chứa & 3 & $\mathrm{~L}$ \\
Công suất máy bơm & 18 & $\mathrm{~W}$ \\
Tốc độ bơm tối đa & 8 & $\mathrm{~L} / \mathrm{min}$ \\
Tốc độ valve tiết lưu & 0,7 & $\mathrm{~m}^{3} / \mathrm{h}$ \\
Cảm biến lưu lượng & $0,5-3$ & $\mathrm{~L} / \mathrm{min}$ \\
Kích thước (D x R x C) & $600 \times 440 \times 560 \mathrm{~mm}$ \\
Trọng lượng & 19 & $\mathrm{~kg}$ \\
\hline
\end{tabular}

\subsection{Thiết kế bộ điều khiển}

\subsection{1. Điều khiển trươt truyền thống}

Một cách tổng quát, xét một hệ thống phi tuyến bậc hai như sau:

Một cách tổng quát, xét một hệ thống phi tuyến bậc hai như sau:

$$
\ddot{\theta}=f(\theta, \dot{\theta})+g(\theta, \dot{\theta}) u+d(t)
$$

Trong đó, $f(\theta, \dot{\theta})$ và $g(\theta, \dot{\theta})$ là các hàm phi tuyến, $u \in R$ và $\theta \in R$ lần lượt là ngõ vào và ngõ ra của hệ thống, $d(t)$ là nhiễu tác động từ bên ngoài và bị chặn bởi $|d(t)| \leq D$. là:

Gọi tín hiệu tham khảo là $\theta_{d}$ thì sai số hệ thống

$$
e=\theta_{d}-\theta
$$

Hàm trượt được định nghĩa sao cho hệ kín ổn định và đáp ứng của hệ bám theo tín hiệu tham khảo. Hàm trượt được thiết kế như sau:

$$
s=\dot{e}+\lambda e
$$

Với $\lambda$ là hằng số dương.

Thay (2) vào (3), hàm trượt được viết lại như sau:

$$
s=\left(\dot{\theta}_{d}-\dot{\theta}\right)+\lambda\left(\theta_{d}-\theta\right)
$$

Lấy đạo hàm của $s$ và thay $\ddot{\theta}$ từ (1) vào, thu được biểu thức như sau:

$$
\dot{s}=\ddot{\theta}_{d}-\ddot{\theta}+\lambda\left(\dot{\theta}_{d}-\dot{\theta}\right)=\ddot{\theta}_{d}-f-g u-
$$

$d(t)+\lambda\left(\dot{\theta}_{d}-\dot{\theta}\right)$.

Theo lý thuyết ổn định Lyapunov, chọn một hàm xác định dương:

$$
V=\frac{1}{2} s^{2}
$$

Từ phương trình (6) suy ra:

$$
\dot{V}=s . \dot{S}
$$

Để $\dot{V}$ xác định âm, chọn $\dot{s}=-k \operatorname{sgn}(s)$, thay $\dot{s}$ vào $(7)$ được:

$$
\dot{V}=-s k \operatorname{sgn}(s)
$$

với $k$ là hằng số dương chọn trước. Từ $(8)$ ta thấy, nếu $s>0$ thì $\operatorname{sgn}(s)=1 \rightarrow \dot{V}<0$, nếu $s<0$ thì 
$\operatorname{sgn}(s)=-1 \rightarrow \dot{V}<0$ và nếu $s=0$ thì $\operatorname{sgn}(s)=0 \rightarrow$ $\dot{V}=0$. Tức là, khi $\rightarrow \propto \propto$ thì $\dot{V}<0$, chứng tỏ hệ thống sẽ ổn định theo tiêu chuẩn Lyapunov.

Như vậy, luật điều khiển của bộ điều khiển trượt được chọn như sau:

$$
u_{S M C}=\frac{1}{g}\left[-f+\ddot{\theta}_{d}+\lambda \dot{e}+k \operatorname{sgn}(s)\right]
$$

\subsection{2. Điều khiển truợt thich nghi dùng mạng neuron $R B F$}

Từ phương trình tổng quát của hệ thống (1), ta thấy trong thực tế các hàm $f(\theta, \dot{\theta})$ và $g(\theta, \dot{\theta})$ thường là các hàm phi tuyến và chưa biết trước. Vì vậy, việc thiết kế luật trượt truyền thống sẽ gặp khó khăn. Nghiên cứu này sử dụng hai mạng neuron RBF để xấp xỉ các hàm $f(\theta, \dot{\theta})$ và $g(\theta, \dot{\theta})$ trong luật trượt (9). Cả hai mạng neuron RBF này đều có cấu trúc [2-5-1], với 2 nút vào, 5 nút Gauss và 1 nút ra được trình bày trên Hình 2.
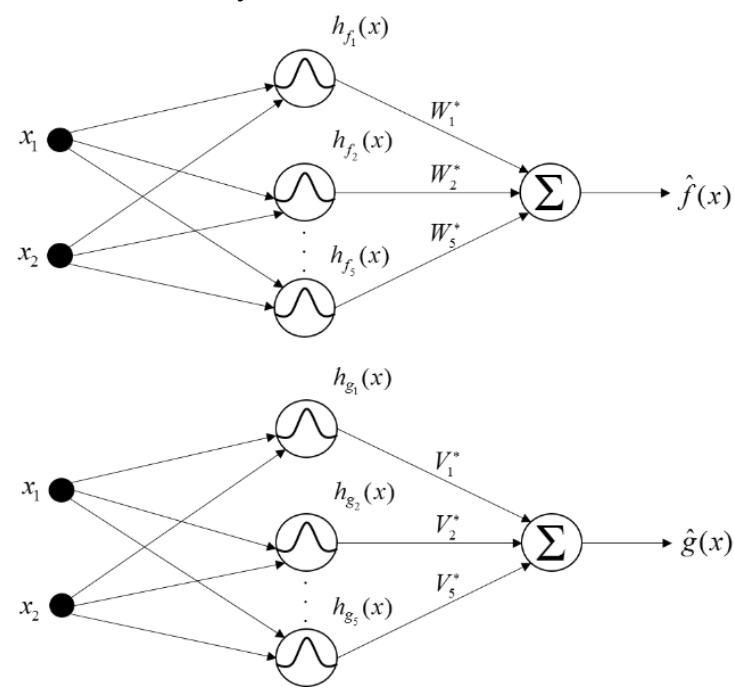

Hình 2: Cấu trúc mạng neuron RBF

Thuật toán dùng mạng neuron RBF để nhận dạng hai hàm $\hat{f}(x)$ và $\hat{g}(x)$ là:

$$
\begin{gathered}
h_{f \mid g_{j}}=\exp \left(\frac{\left\|x-c_{j}\right\|^{2}}{2 b_{j}^{2}}\right) \\
\hat{f}(x)=W^{* T} h_{f_{j}}(x)+\varepsilon_{f} \\
\hat{g}(x)=V^{* T} h_{g_{j}}(x)+\varepsilon_{g}
\end{gathered}
$$

Trong đó, $\boldsymbol{x}$ là ngõ vào của mạng; $j$ là số lớp ẩn của mạng; $h_{f \mid g_{j}}$ là ngõ ra của các hàm Gauss; $\boldsymbol{W}^{*}$ và $V^{*}$ là ma trận trọng số; $\varepsilon_{f}$ và $\varepsilon_{g}$ là sai số xấp xỉ của mạng; $\hat{f}(x)$ và $\hat{g}(x)$ là các ngõ ra xấp xỉ của mạng.
Ngõ vào của mạng được chọn là véc tơ $\boldsymbol{x}=$ $\left[x_{1} x_{2}\right]^{T}=[e \dot{e}]^{T}$, số hàm Gauss $j=5$ và nếu bỏ qua sai số xấp xỉ thì ngõ ra của mạng neuron RBF là:

$$
\hat{f}(x)=W^{* T} h_{f}(x), \quad \hat{g}(x)=V^{* T} h_{g}(x)
$$
Gauss.

Trong đó, $h_{f}(x)$ và $h_{g}(x)$ là ngõ ra các hàm

Khi đã ước lượng được $\hat{f}(x)$ và $\hat{g}(x)$ tín hiệu điều khiển (9) được thay thế thành:

$$
u_{S M C}=\frac{1}{\hat{g}(x)}\left[-\hat{f}(x)+\ddot{\theta}_{d}+\lambda \dot{e}+k \operatorname{sgn}(s)\right]
$$

Thay (12) vào (5), ta có:

$$
\begin{aligned}
\dot{s} & =\ddot{\theta}_{d}-f-\hat{g} u+(\hat{g}-g) u-d(t)+\lambda \dot{e} \\
& =\ddot{\theta}_{d}-f-\hat{g} \frac{1}{\hat{g}(x)}\left[-\hat{f}(x)+\ddot{\theta}_{d}+\lambda \dot{e}+\right.
\end{aligned}
$$

$k \operatorname{sgn}(s)]+(\hat{g}-g) u-d(t)+\lambda \dot{e}$

$$
\begin{aligned}
& =(\hat{f}-f)-k \operatorname{sgn}(s)+(\hat{g}-g) u- \\
& d(t)=\tilde{f}-k \operatorname{sgn}(s)+\tilde{g} u-d(t) \\
& =\widetilde{W}^{T} h_{f}(x)-\varepsilon_{f}-k \operatorname{sgn}(s)+ \\
& \left(\tilde{V}^{T} h_{g}(x)-\varepsilon_{g}\right) u-d(t) \text {. } \\
& \text { với: } \widetilde{W}=W^{*}-\widehat{W}, \tilde{V}=V^{*}-\widehat{V} \text {. Và: } \\
& \tilde{f}=\hat{f}-f=\widehat{W}^{T} h_{f}(x)-W^{* T} h_{f}(x)- \\
& \varepsilon_{f}=\widetilde{W}^{T} h_{f}(x)-\varepsilon_{f} \\
& \tilde{g}=\hat{g}-g=V^{T} h_{g}(x)-V^{* T} h_{g}(x)-\varepsilon_{g}= \\
& \tilde{V}^{T} h_{g}(x)-\varepsilon_{g}
\end{aligned}
$$

Theo lý thuyết Lyapunov, hàm xác định dương được chọn như sau (Ye et al., 2020; Miqoi et al., 2019):

$$
L=\frac{1}{2} s^{2}+\frac{1}{2 \gamma_{1}} \widetilde{W}^{T} \widetilde{W}+\frac{1}{2 \gamma_{2}} \tilde{V}^{T} \tilde{V}(8)
$$

Với $\gamma_{1}>0$ và $\gamma_{2}>0$.

Lấy đạo hàm hai vế (16) và thayṡcủa (15) vào, thu được:

$$
\begin{gathered}
\dot{L}=s \dot{s}+\frac{1}{\gamma_{1}} \widetilde{W}^{T} \dot{\widetilde{W}}+\frac{1}{\gamma_{2}} \tilde{V}^{T} \dot{\tilde{V}} \\
=s\left(\widetilde{W}^{T} h_{f}(x)-\varepsilon_{f}-k \operatorname{sgn}(s)+\right. \\
\left.\left(\tilde{V}^{T} h_{g}(x)-\varepsilon_{g}\right) u-d(t)\right)-\frac{1}{\gamma_{1}} \widetilde{W}^{T} \dot{\hat{W}}-\frac{1}{\gamma_{2}} \tilde{V}^{T} \dot{\hat{V}}(10) \\
=\widetilde{W}^{T}\left(\operatorname{sh} h_{f}(x)-\frac{1}{\gamma_{1}} \dot{\hat{W}}\right)+\tilde{V}^{T}\left(s h_{g}(x) u-\right. \\
\left.\frac{1}{\gamma_{2}} \dot{\hat{V}}\right)+s\left(-\varepsilon_{f}-k \operatorname{sgn}(s)-\varepsilon_{g} u-d(t)\right)
\end{gathered}
$$

Luật thích nghi được thiết kế như sau: 


$$
\begin{gathered}
\dot{\hat{W}}=-\gamma_{1} \operatorname{sh}_{f}(x) \\
\dot{\hat{V}}=-\gamma_{2} \operatorname{sh}_{g}(x) u
\end{gathered}
$$

Thay $\dot{W}$ và $\dot{V}$ từ (20) vào (19) thu được:

$$
\begin{aligned}
\dot{L} & =s\left(-\varepsilon_{f}-k \operatorname{sgn}(s)-\varepsilon_{g} u-d(t)\right) \\
& =\left(-\varepsilon_{f}-\varepsilon_{g} u-d(t)\right) s-\operatorname{sk} \operatorname{sgn}(s)
\end{aligned}
$$

Do sai số xấp xỉ $\varepsilon_{f}$ và $\varepsilon_{g}$ đủ nhỏ, nên ta có thể chọn $\left(-\varepsilon_{f}-\varepsilon_{g} u-d(t)\right)=0$. Khi đó, phương trình (22) được viết lại như sau:

$$
\dot{L}=-\operatorname{sk} \operatorname{sgn}(s)
$$

Với $k$ là hằng số dương chọn trước.

Phương trình (23) cho thấy nếu $s>0$ thì $\dot{L}<0$; nếu $s<0$ thì $\dot{L}<0$ và nếu $s=0$ thì $\dot{L}=0$, chứng tỏ hệ thống sẽ ổn định theo tiêu chuẩn Lyapunov.

\section{Bảng 2: Ảnh hưởng của việc tăng $\left\{K_{p}, K_{i}\right\}$ đối với đáp ứng của hệ thống điều khiển}

\begin{tabular}{lllll}
\hline Tăng độ lợi & Thời gian tăng & Độ vọt lố & Thời gian xác lập & Sai số xác lập \\
\hline$K_{p}$ & Giảm & Tăng & Thay đồi ít & Giảm \\
$K_{i}$ & Giảm & Tăng & Tăng & Triệt tiêu \\
\hline
\end{tabular}

Bộ điều khiển PI áp dụng cho thiết bị RT020 được Gunt (2021) tích hợp sẵn trên giao diện Hình 3. Phần mềm này có thể kiểm soát 6 thiết bị, từ RT010 đến RT060. Trong nghiên cứu này, mô hình được chọn để thực nghiệm là hệ ổn định lưu lượng chất lỏng - RT020. Bộ điều khiển PI được chạy với bộ thông số mặc định $K_{p}=0,01$ và $T_{n}=0,5$

\subsection{3. Điều khiển kết hơp PI-SMC}

Bộ điều khiển tích phân tỷ lệ PI theo (24) được áp dụng nhiều trong công nghiệp, nhờ hiệu quả đáp ứng và tính đơn giản của nó (Huang, H. P. \& Jeng, J. C., 2005).

$$
\begin{gathered}
u_{P I}=K_{p} e(t)+K_{i} \int_{0}^{t} e(t) d t \\
e(t)=X_{r e f}(t)-X(t)
\end{gathered}
$$

Trong đó, $K_{p}$ và $K_{i}$ lần lượt là độ lợi tỉ lệ và độ lợi tích phân của bộ điều khiển; $X_{\text {ref }}(t)$ là tín hiệu tham khảo và $X(t)$ là đáp ứng của đối tượng. Nhiệm vụ của người thiết kế bộ điểu khiển PI là chọn lựa bộ giá trị $\left\{K_{p}, K_{i}\right\}$ thỏa mãn các yêu cầu về chất lượng điều khiển. Việc tăng các thông số $\left\{K_{p}, K_{i}\right\}$ ảnh hưởng đến thời gian tăng (rise time), độ vọt lố (overshoot) và thời gian xác lập (settling time) của đáp ứng, được cho trong Bảng 2 (Kanagaraj et. al., 2008).

nhằm duy trì lưu lượng chất lỏng tại 120 1/h, kết quả như Hình 4. Lưu ý, thời hằng tích phân $T_{n}$ trên Hình 4 quan hệ với thông số $K_{i}$ như sau: $K_{i}=K_{p} / T_{n}=$ $0,01 / 0,5=0,02$. Kết quả Hình 4 cho thấy ngõ ra lưu lượng $X$ (đáp ứng) bị vọt lố hơn $13,33 \%$ so với lưu lượng tham khảo $W$, đồng thời lưu lượng ngõ ra $X$ bị dao động nhiều, khi tín hiệu điều khiển $Y$ bị gợn sóng.

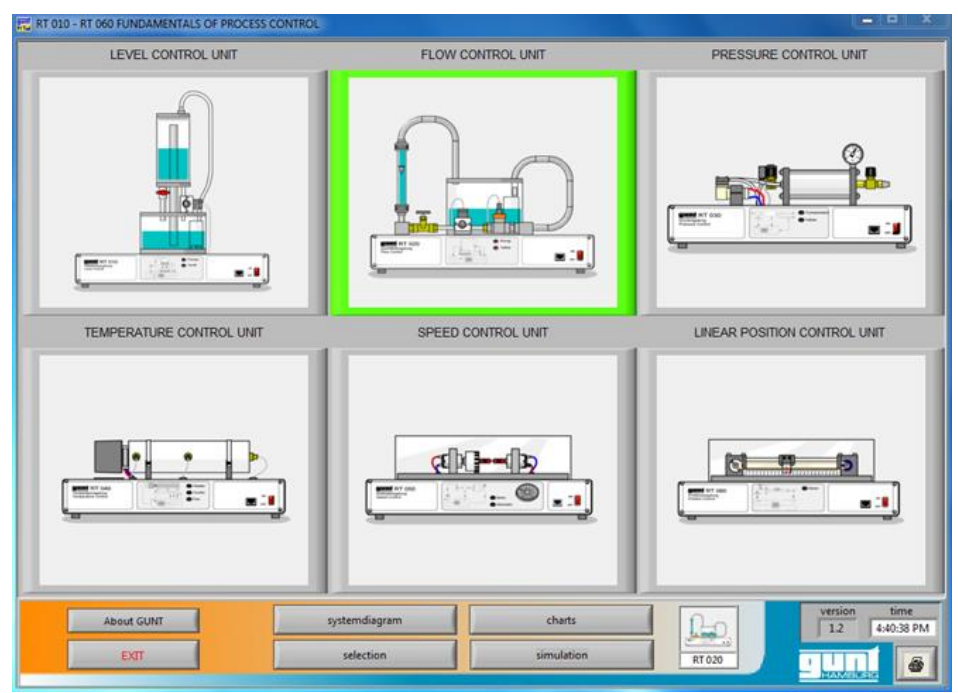

Hình 3: Các mô hình điều khiển của Gunt 


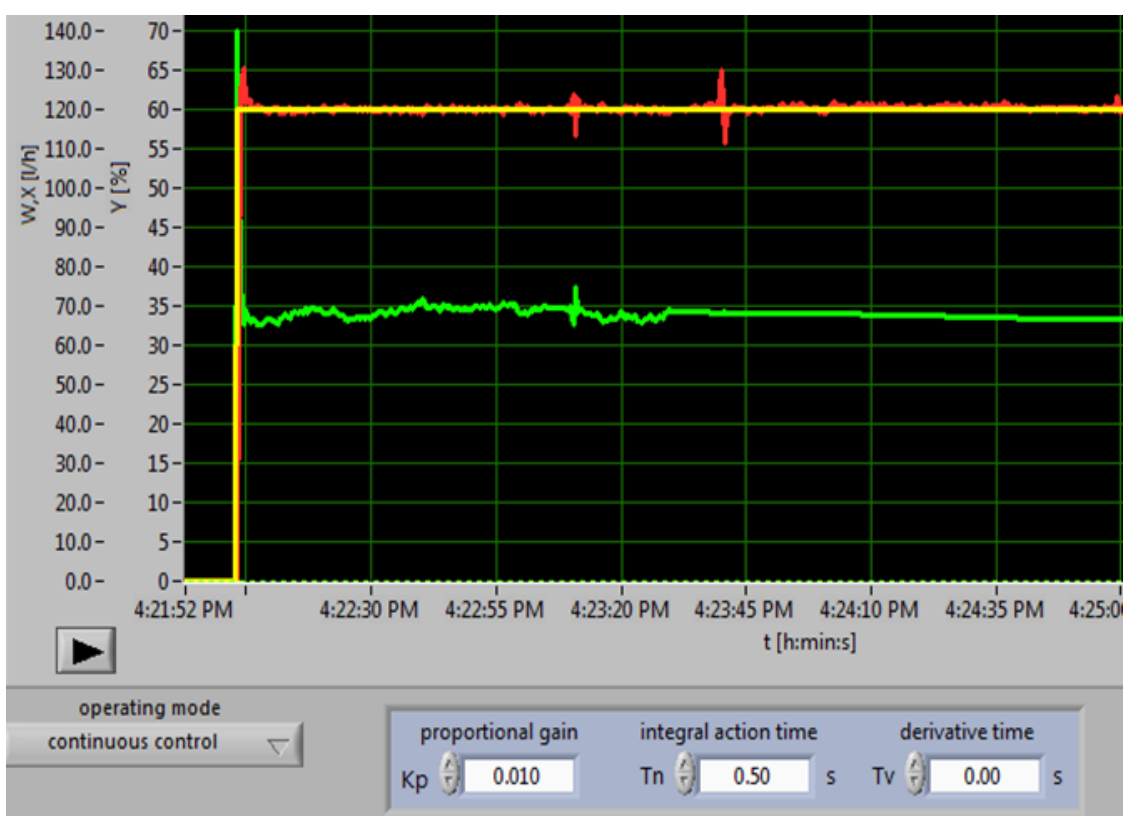

Hình 4: Kết quả điều khiển PI hệ RT020 trên phần mềm Gunt

Như đã đề cập, với bộ tham số cố định, bộ điều khiển PID nói chung và bộ điều khiển PI nói riêng, hoạt động kém hiệu quả, khi có sự biến động đối tượng hay điều kiện thực tế. Vì thế, để đảm bảo chất lượng điều khiển, cần có thêm một cơ chế cùng tác động, có khả năng thích ứng với sự biến đổi này. Việc kết hợp giữa bộ điều khiển PI truyền thống và bộ SMC thích nghi, gọi tắt là bộ điều khiển PI-SMC là một lựa chọn nhằm khắc phục vấn đề này. Bộ điều khiển đề xuất có cấu trúc như Hình 5 . Với cấu trúc này, tín hiệu điều khiển là:

$$
u=u_{S M C}+u_{P I}
$$

Trong đó, $u_{S M C}$ là tín hiệu điều khiển trượt thích nghi và $u_{P I}$ là tín hiệu điều khiển PI.

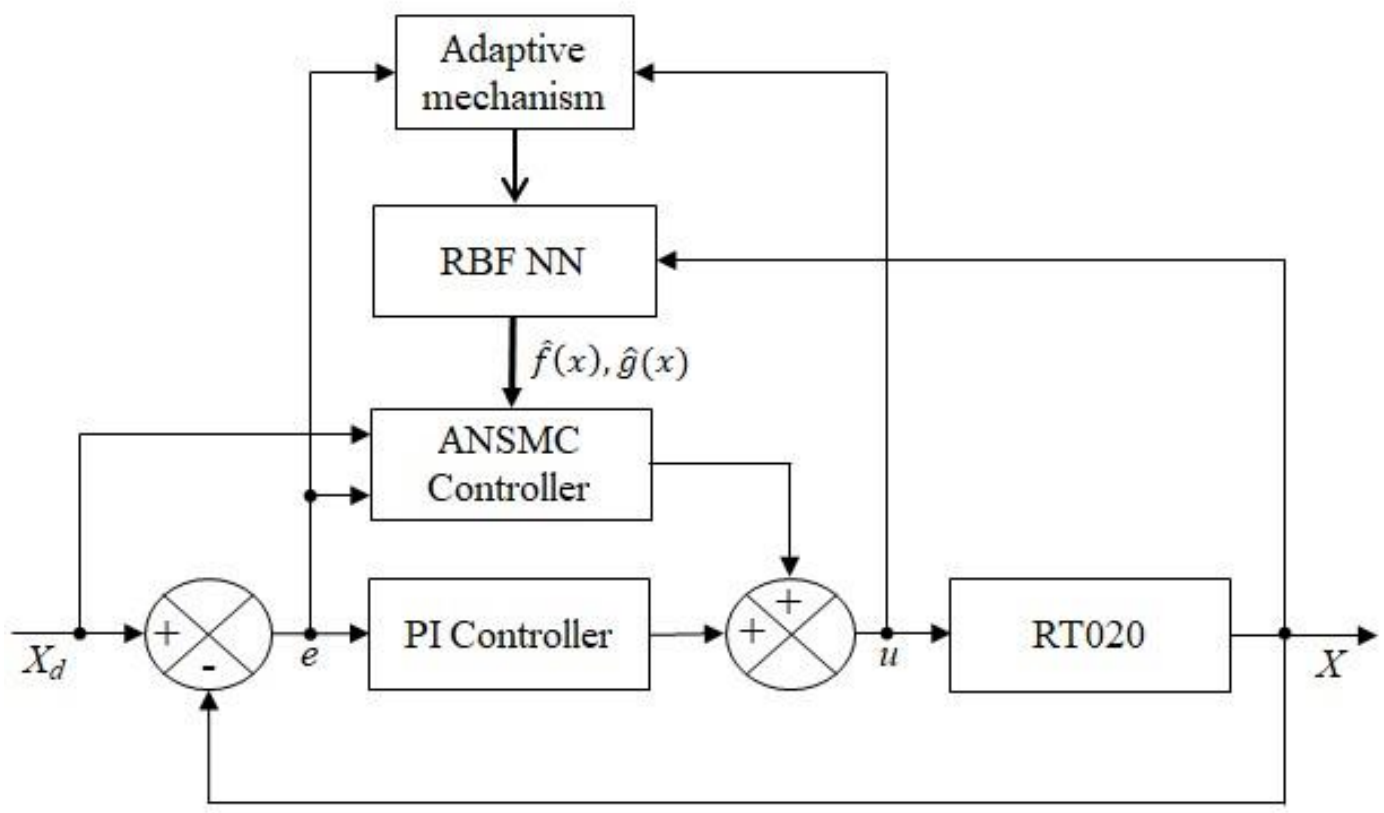

Hình 5: Nguyên lý điều khiển kết họ̣p PI-SMC 


\section{KẾT QUẢ VÀ THẢO LUẬN}

Dựa trên sơ đồ Hình 5, mô hình thực nghiệm điều khiển kết hợp PI-SMC được xây dựng trên MATLAB/Simulink, được trình bày như Hình 6 .

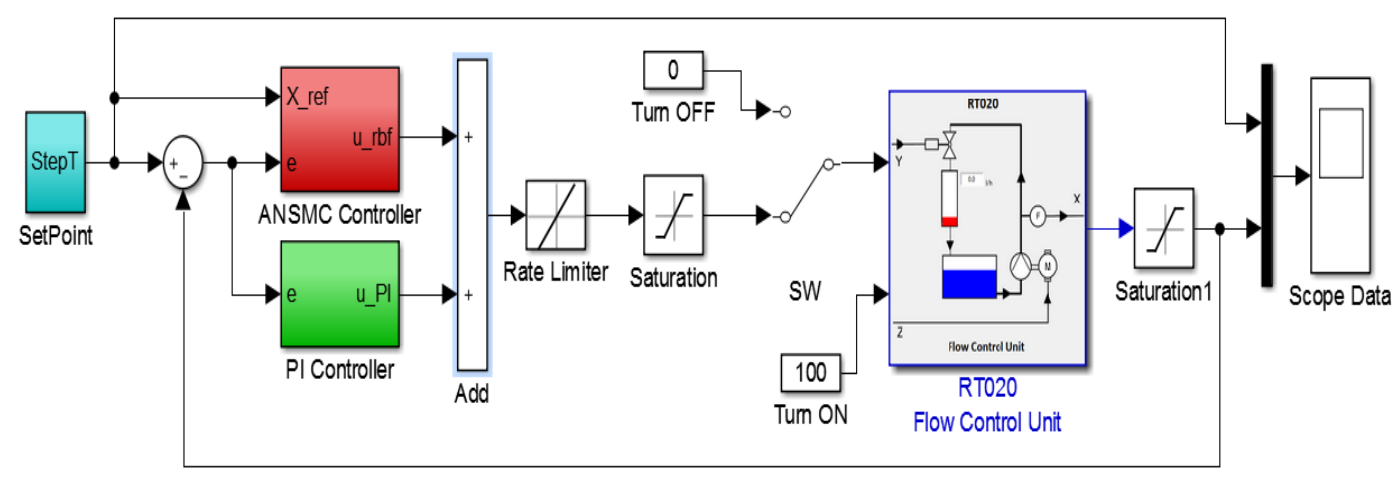

Hình 4: Bộ điều khiển kết hợp PI-SMC thực nghiệm

Thực nghiệm thứ nhất được tiến hành với thời gian $t=40 \mathrm{~s}$, tốc độ lấy mẫu là $T s=0,1 \mathrm{~s}$, thông số của bộ điều khiển PI mặc định là $K_{p}=0,01$ và $K_{i}=$ 0,02 (hay $T_{n}=0,5$ ), thông số của bộ SMC thích nghi được khởi tạo là $b_{j}=5, \quad c_{i j}=$ $\left[\begin{array}{lllll}-5 & -2,5 & 0 & 2,5 & 5 \\ -5 & -2,5 & 0 & 2,5 & 5\end{array}\right], W^{*}$ và $V^{*}$ ngẫu nhiên $\epsilon$ $(0,1)$ và hệ số mặt trượt trong $(3)$ được chọn $\lambda=1$. Đáp ứng của hệ RT020 được trình bày ở Hình 7 . Kết quả này cho thấy đáp ứng lưu lượng của hệ RT020 bám được lưu lượng tham khảo. Tuy nhiên, chất lượng điều khiển chưa được tốt tại các thời điểm lưu lượng tham khảo thay đổi đột ngột theo hàm nấc. Điều này cho thấy, thực tế việc chọn lựa các giá trị khởi tạo của mạng neuron RBF và hệ số $\lambda$ của mặt trượt có tác động đến chất lượng điều khiển.

Do vậy, các ở các thực nghiệm tiếp theo, các tham số trên được thay đổi và dựa vào một số tiêu chí cơ bản để đánh giá chất lượng điều khiển. Hai tiêu chí được sử dụng gồm IAE (Integral of the Absolute value of the Error) và IATE (Integral of the time weighted Absolute value of the Error), định nghĩa bởi (26) và (27):

$$
\begin{aligned}
& \text { IAE }=\int_{0}^{\infty}|e(t)| \mathrm{dt} \\
& \text { IATE }=\int_{0}^{\infty} t|e(t)| d t
\end{aligned}
$$

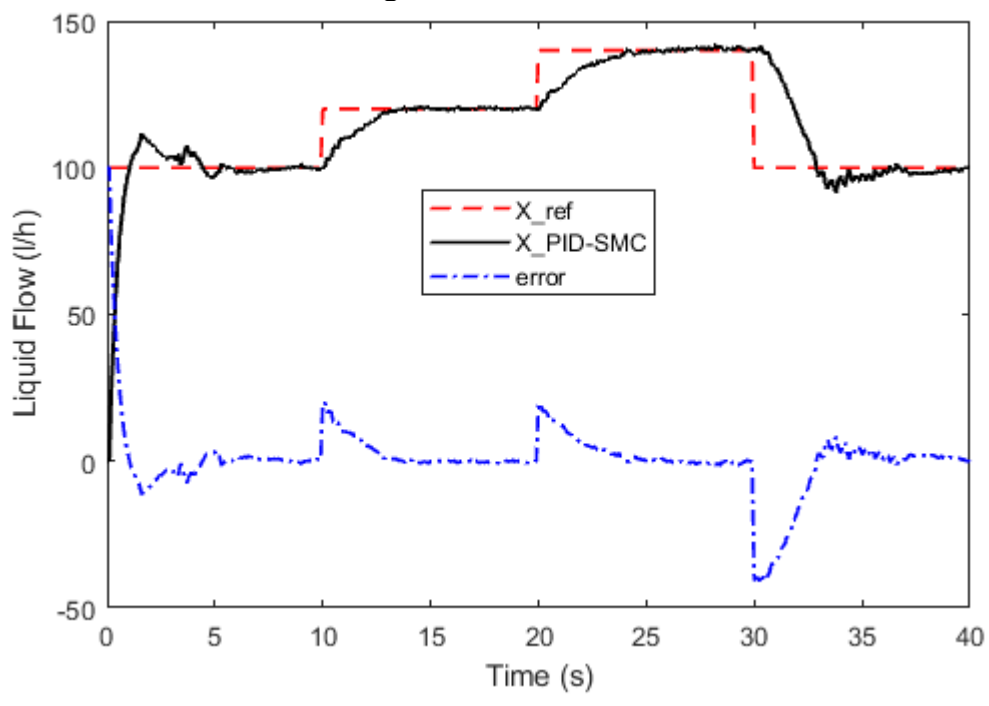

Hình 5: Kết quả thực nghiệm 1 bộ điều khiển kết họ̣p PI-SMC

Bảng 3, Bảng 4, Bảng 5 và Bảng 6 trình bày kết quả của 20 thực nghiệm khác nhau, mà mỗi thực nghiệm các tiêu chí IAE và IATE tương ứng được tính toán, nhằm so sánh và chọn lựa bộ tham số tốt nhất cho hệ điều khiển. 
Bảng 3: Cố định $b_{j}, \lambda$ và thay đổi $c_{i j}$

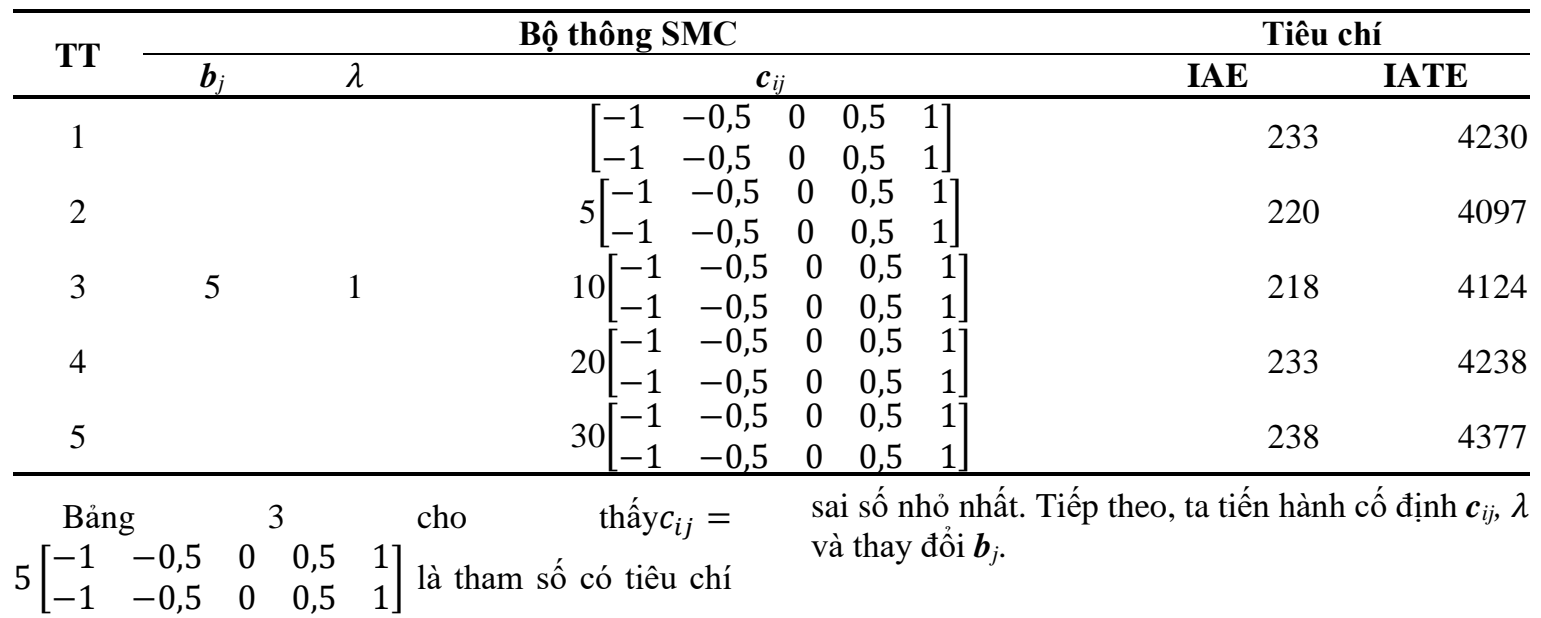

Bảng 4: Cố định $c_{i j}, \lambda$ và thay đổi $b_{j}$

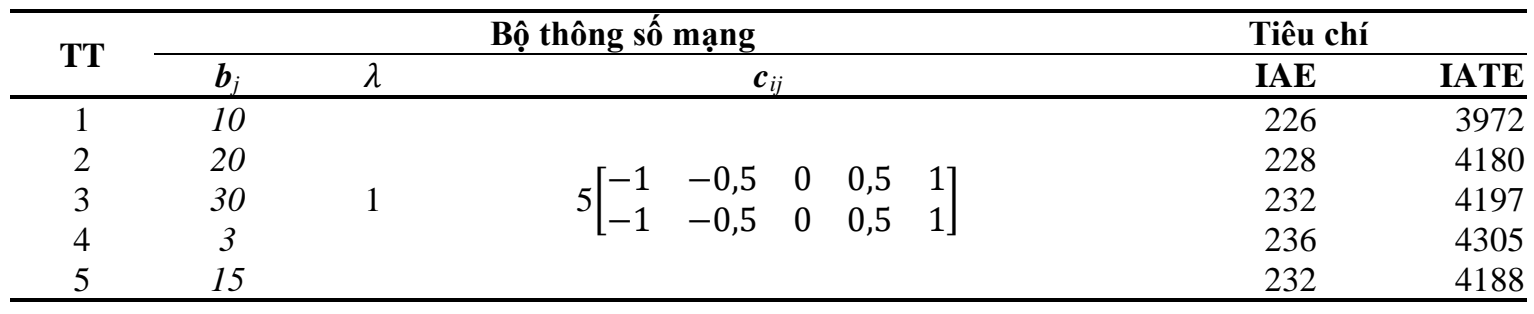

Bảng 4 cho thấy $b_{j}=20$ là bộ tham số có tiêu chí sai số nhỏ nhất. Tiếp theo, ta tiến hành cố định $\boldsymbol{b}_{j}, \boldsymbol{c}_{i j}$ và thay đổi $\lambda$.
Bảng 5 cho thấy $\lambda=5$ là tham số có tiêu chí sai số nhỏ nhất. Tiếp theo, ta thử tiến hành thay đổi đồng thời bộ tham số $\boldsymbol{b}_{j}, \boldsymbol{c}_{i j}$ và $\lambda$.

Bảng 5: Cố định $b_{j}, c_{i j}$ và thay đổi $\lambda$

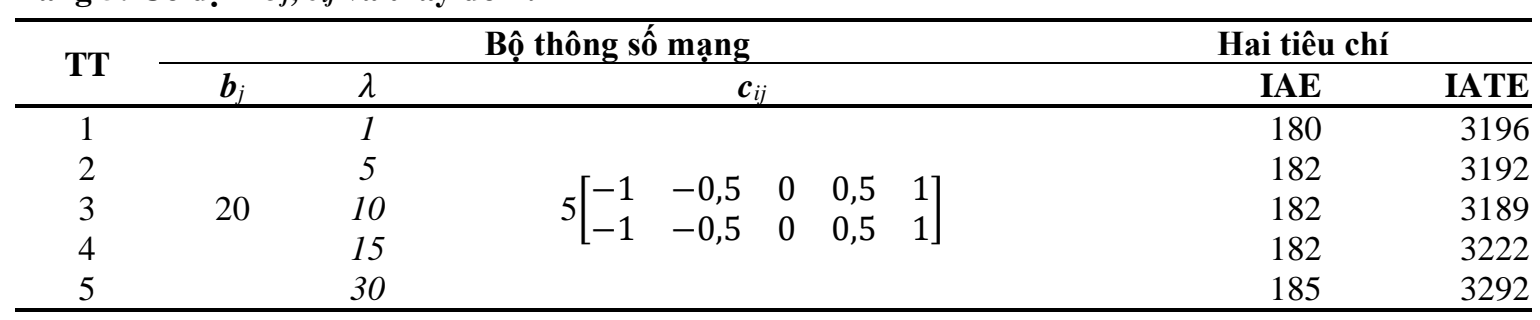

Bảng 6: Đồng thời thay đổi cả 3 thông số

\begin{tabular}{|c|c|c|c|c|c|c|c|c|c|}
\hline \multirow{2}{*}{ TT } & \multicolumn{7}{|c|}{ Bộ thông số mạng } & \multicolumn{2}{|c|}{ Hai tiêu chí } \\
\hline & $\boldsymbol{b}_{j}$ & $\lambda$ & \multicolumn{5}{|c|}{$c_{i j}$} & IAE & IATE \\
\hline \multirow{2}{*}{1} & 15 & l & & $\overline{-1}$ & $-0,5$ & $\overline{0}$ & $\begin{array}{ll}0,5 & 11\end{array}$ & \multirow{2}{*}{183} & \multirow{2}{*}{3234} \\
\hline & 15 & $I$ & 15 & -1 & $-0,5$ & 0 & $0,5 \quad 1]$ & & \\
\hline \multirow[t]{2}{*}{2} & 15 & 5 & & -1 & $-0,5$ & 0 & $0,5 \quad 1]$ & \multirow{2}{*}{176} & \multirow{2}{*}{3192} \\
\hline & & & & -1 & $-0,5$ & 0 & $0,5 \quad 1]$ & & \\
\hline 3 & 20 & 50 & 20 & $\begin{array}{l}-1 \\
-1 \\
-1\end{array}$ & $-0,5$ & 0 & $\begin{array}{ll}0,5 & 1 \\
0,5 & 1\end{array}$ & 184 & 3181 \\
\hline \multirow{2}{*}{4} & 30 & 30 & 30 & -1 & $-0,5$ & 0 & $0,5 \quad 11$ & \multirow{2}{*}{192} & \multirow{2}{*}{3297} \\
\hline & 30 & 30 & 30 & -1 & $-0,5$ & 0 & $0,5 \quad 1]$ & & \\
\hline 5 & 13 & 13 & 13 & -1 & $-0,5$ & 0 & $0,5 \quad 1]$ & 183 & 3263 \\
\hline
\end{tabular}


Nhận xét: Qua nhiều lần kiểm nghiệm từ Bảng 3 đến Bảng 6 có thể thấy rằng bộ thông số cho kết quả tốt nhất là: $\boldsymbol{b}_{j}=15, \quad \boldsymbol{c}_{i j}=$ $15\left[\begin{array}{lllll}-1 & -0,5 & 0 & 0,5 & 1 \\ -1 & -0,5 & 0 & 0,5 & 1\end{array}\right]$ và $\lambda=5$ với kết quả đáp ứng lưu lượng được trình bày ở Hình 8 . Các thực nghiệm này chưa thể xác định được giá trị tối ưu của bộ tham số $\left(\boldsymbol{b}_{j}, \boldsymbol{c}_{i j}, \lambda\right)$, nhưng nó cho ta nhìn nhận một cách tổng quát về ảnh hưởng thực tế của việc khởi tạo bộ tham số này lên chất lượng điều khiển. Có thể thấy rằng, chỉ thông qua thực nghiệm mới có thể đánh giá được tổng thể về tác động của các tham số khởi tạo của bộ điều khiển, điều mà hầu hết các công bố thường không trình bày một cách chi tiết.

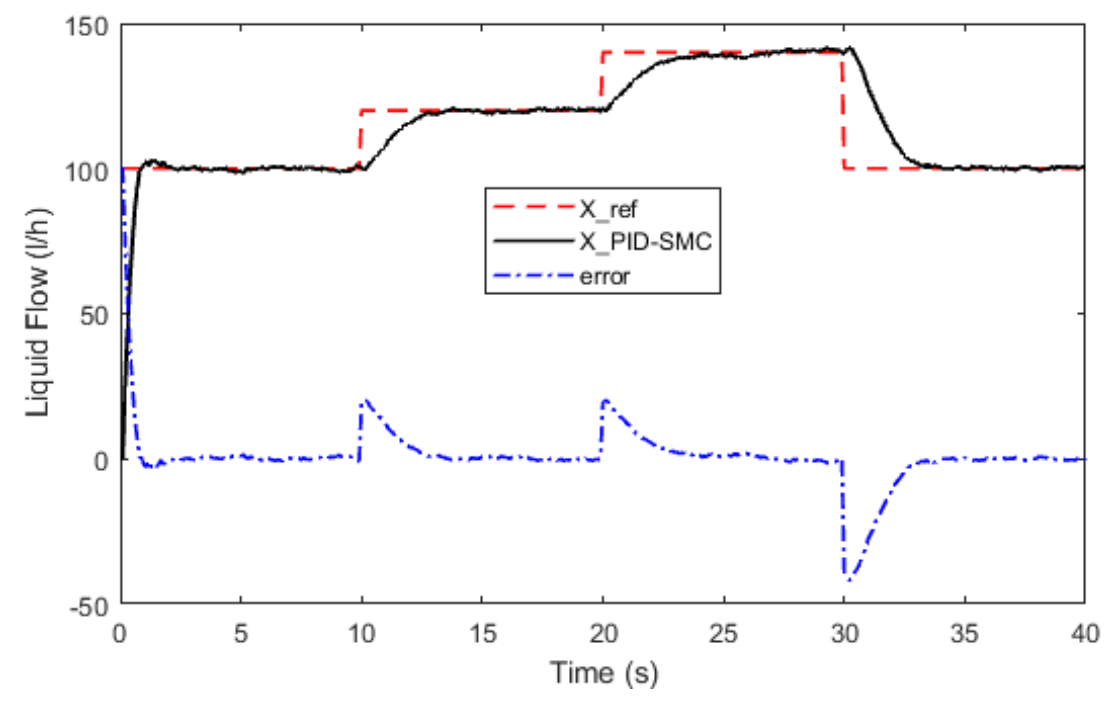

Hình 6: Kết quả thực nghiệm cho đáp ứng tốt nhất

Kết quả thực nghiệm trên Hình 8 có các tiêu chí $\mathrm{IAE}=176$ và $\mathrm{IATE}=3192$ là cặp tiêu chí có giá trị sai số nhỏ nhất. Ứng với bộ tham số khởi tạo $\boldsymbol{b}_{j}=15$, $c_{i j}=15[$ [. ] và $\lambda=5$, bộ điề̀u khiển kết hợp PI-SMC được so sánh với bộ điều khiển mặc định PI trên hệ RT020, cho kết quả hiển thị cận cảnh khoảng thời gian quá độ, được minh họa trên Hình 9.

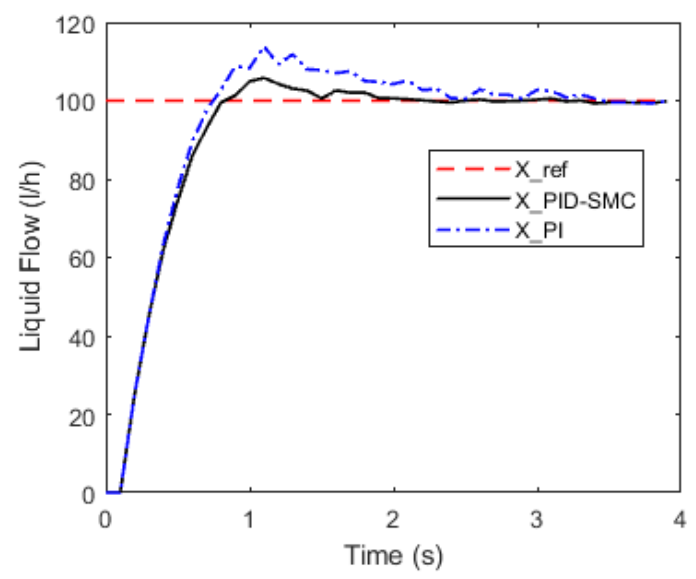

Hình 7: So sánh kết quả thực nghiệm giữa bộ điều khiển PI-SMC và PI

Tại bộ tham số này, đáp ứng lưu lượng hệ RT020 được cải thiện đáng kể so với khi áp dụng bộ điều khiển PI mặc định, với độ vọt lố nhỏ hơn $5 \%$, thời gian xác lập nhỏ hơn 2 giây và sai số xác lập nhỏ hơn $0,3 \mathrm{l} / \mathrm{h}$.

\section{KẾT LUẬN VÀ ĐỀ NGH!}

Giải pháp kết hợp bộ điều khiển PI truyền thống và bộ điểu khiển trượt thích nghi dùng mạng neuron RBF đã được để xuất và kiểm nghiệm trên hệ ổn định lưu lượng chất lỏng RT020 của hãng GuntHamburg. Kết quả thực nghiệm chứng tỏ rằng việc chọn lựa giá trị khởi tạo của mạng neuron RBF gồm ma trận tâm và độ rộng của các hàm Gauss, cùng với hệ số mặt trượt có ảnh hưởng lớn đến chất lượng điều khiển, thể hiện thông qua các tiêu chuẩn chất lượng IAE và IATE. Quá trình thực nghiệm cho thấy cơ chế trượt thích nghi hoàn toàn có thể sử dụng để khắc phục hạn chế về việc cố định tham số của bộ điều khiển PI truyền thống. Với giá trị khởi tạo của bộ tham số được chọn, bộ điều khiển kết hợp PISMC đã cải thiện tốt đáp ứng lưu lượng trên hệ RT020, với độ vọt lố thấp hơn 5\%, thời gian xác lập nhỏ hơn 2 giây và sai số xác lập nhỏ hơn 0,3 lít/giờ. Kỹ thuật điều khiển này hoàn toàn có thể áp dụng cho các đối tượng phi tuyến khác mà ở đó bộ điều khiển PID truyền thống với tham số cố định không đáp ứng được yêu cầu về chất lượng điều khiển. 


\section{TÀI LIỆU THAM KHẢO}

Nguyễn Hoàng Dũng \& Dương Hoài Nghĩa. (2010). Điều khiển trượt thích nghi dùng mô hình nơron mờ. Tạp chí Khoa học Truờng Đại học Cần Tho, 15a, 273-282.

Nguyễn Hoàng Dũng. (2012). Điều khiển trượt dựa trên hàm trượt kiểu PID. Tạp chi Khoa học Truò̀ng Đại học Cần Tho, 21a, 30-36.

Gopika, K. \& Ms. Latha. V. (2016). Sliding Mode based Level Controller with First Order Plus Delay Time (FOPDT) Modeling. Inter. J. of Engineering Research \& Technology (IJERT), 4(17), 1-5.

Gunt Gerätebau GmbH. (2004). Experiment Instructions - RT010- RT060 Principles of Control Engineering. Barsbüttel Germany, Publication-no: 918.00000 A 0X0 02 (A).

Huang, H. P. \& Jeng, J. C. (2005). Process Reaction Curve and Relay Methods Identification and PID Tuning. In: Johnson M.A., Moradi M.H. (eds) PID Control. Springer, London. https://doi.org/10.1007/1-84628-148-2_8.

Keshtkar, S., Keshtkar, J. \& Poznyak, A. (2016). Adaptive sliding mode control for solar tracker orientation. In: 2016 American Control Conference-ACC, 6543-6548, IEEE. DOI: 10.1109/ACC.2016.7526700

Kanagaraj, N., Sivashanmugam, P. \& Paramasivam, S. (2008). Fuzzy Coordinated PI Controller: Application to the Real-Time Pressure Control
Process. Advances in Fuzzy Systems, 2008, Article ID 691808, 1-

9. https://doi.org/10.1155/2008/691808.

LabJack Corporation. (2003). LabJack U12

Quickstart Guide. Revision 1.09, www.LabJack.com.

Nguyễn Đức Minh, Dương Hoài Nghĩa \& Nguyễn Đức Thành. (2009). Điều khiển trượt thích nghi dùng mạng nơ-ron. Tạp chi Khoa hoc \& Công nghệ các truò̀ng đại học kỹ thuật, 71, 1-5.

Miqoi, S., Ougli, A. E. \& Tidhaf, B. (2019). Design of an adaptive sliding mode controller for efficiency improvement of the MPPT for PV water pumping. International Journal of Intelligent Engineering Informatics, 7(1), 19-36. https://doi.org/10.1504/IJIEI.2019.097550

Nguyễn Chí Ngôn. (2011). Bộ điều khiển PI mờ: Từ thiết kế đến ứng dụng. Tạp chi Khoa hoc Truờng Đai hoc Cần Tho, 18a, 82-92.

Nguyễn Đình Tứ, Lê Hoàng Đăng, Trần Chí Cường \& Nguyễn Chí Ngôn. (2017). Điều khiển thích nghi theo mô hình tham khảo dựa trên mạng nơron RBF. Tạp chi Khoa họ Trường Đại hoc Cần Tho, 50(A), 37-42.

https://doi.org/10.22144/ctu.jvn.2017.064

Ye, T., Luo, Z. \& Wang, G. (2020). Adaptive sliding mode control of robot based on fuzzy neural network. Journal of Ambient Intelligence and Humanized Computing, 11(12), 6235-6247. https://doi.org/10.1007/s12652-020-01809-2 